\begin{abstract}
We analyze electrostatic interaction between a sharp conducting tip and a thin one-dimensional wire, e.g., a carbon nanotube, in a scanned gate microscopy (SGM) experiment. The problem is analytically tractable if the wire resides on a thin dielectric substrate above a metallic backgate. The characteristic spatial scale of the electrostatic coupling to the tip is equal to its height above the substrate. Numerical simulations indicate that imaging of individual electrons by SGM is possible once the mean electron separation exceeds this scale (typically, a few tens of $\mathrm{nm}$ ). Differences between weakly and strongly invasive SGM regimes are pointed out.
\end{abstract}




\title{
Scanned gate microscopy of a one-dimensional quantum dot
}

\author{
Lingfeng M. Zhang and Michael M. Fogler \\ Department of Physics, University of California San Diego, 9500 Gilman Drive, \\ La Jolla, California 92093
}

July 14, 2018

Scanned gate microscopy (SGM) is a recent addition to the arsenal of modern scanned probe techniques that enable one to study and manipulate nanoscale objects at a single-electron level. Much interest has been attracted by experiments 1, 2, 3, 4, 5, 6] where the SGM has been employed for probing carbon nanotube (CNT) quantum dots under the conditions of Coulomb blockade. In those experiments, a movable sharp tip with a controllable electrostatic potential was employed to add or remove electrons from the dots one by one, which was monitored via transport measurements.

The goal of this work is to explore the capabilities of the SGM for imaging the real-space electron structure in a CNT dot. In parallel, we develop an analytical approach that clarifies what controls the fundamental limits of the SGM spatial resolution. We focus on a typical SGM geometry where the CNT resides on a dielectric substrate above a metallic backgate (Fig. 11). We show below that if the substrate is thin, so that the gate efficiently screens Coulomb interactions, the requisite electrostatic problem is tractable. We find the potential created by the charged tip in terms of elementary functions. For realistic experimental parameters our formula proves to be accurate to about $5 \%$, which enables us to test with confidence the assumptions made about the same quantity in prior literature 4, 5, 7. In particular, we show that for the case of a sharp tip the spatial resolution of the SGM is set by the tip's height above the substrate. For thick substrates, the resolution degrades and becomes of the order of the geometric mean of the tip height and the CNT length. Typical experimental parameters put one in between these two limiting cases, with the net resolution of the order of a few tens of $\mathrm{nm}$. We propose that this may be sufficient for imaging of individual charges in a few-electron quantum dots and support this idea by numerical simulations. Our method of analysis can be extended to other one-dimensional (1D) and quasi-1D systems (multiple quantum dots, CNT networks, etc.), and so we hope it may be a useful tool in the experimental practice of SGM.

We consider a CNT of radius $R$ resting on an insulating substrate of thickness $D$ deposited on a metallic gate (Fig. 1). We assume that the contact resistances are high, so that the CNT behaves as a quantum dot. Such a dot is probed by a movable conducting tip, hovering at the height $h$ above the point $\mathbf{r}_{t}=\left(x_{t}, y_{t}\right)$ of the substrate plane. The radius $w(\zeta)$ of the tip as a function of distance $\zeta=z-h$ from its end is given by 


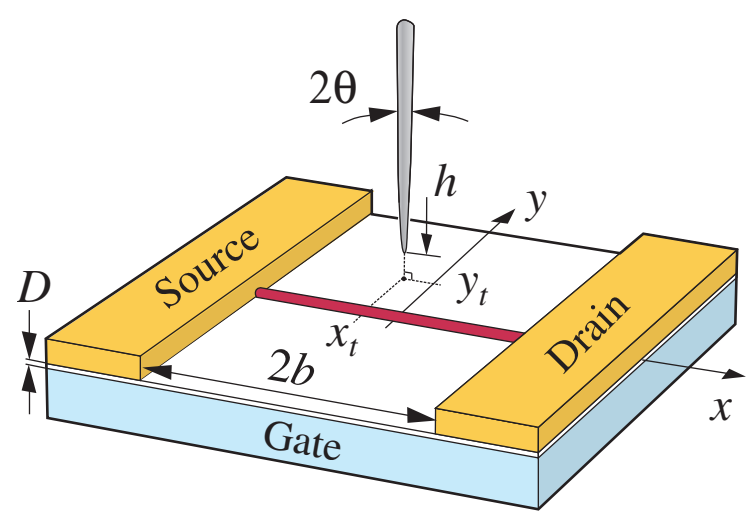

Figure 1: (Color online) The SGM setup studied in this paper: a movable metallic tip that probes a 1D wire (the horizontal cylinder).

$w(\zeta)=\theta \zeta$ at $l \ll \zeta \ll L$, where $\theta \ll 1$ is the apex angle and $L$ is the full length of the tip. Parameter $l \ll h$ accounts for the rounding of the tip's end [8].

Our first task is to analyze Coulomb interactions among the charges on the CNT, gate, and the tip. For simplicity, we set all dielectric constants to unity 9]. We start with a qualitative picture. The excess charge density $\rho_{d}(x)$ induced on the CNT by the external gates can be deduced from classical electrostatics of a thin metallic wire. Earlier microscopic calculations [7, 10, 11, 12] proved that this is a good approximation when lengthscales of interest exceed $e / \rho_{d}$. If the distance $D$ to the screening gate is much smaller than the length of the CNT, $\rho_{d}(x)$ is nearly uniform. This is because the effective interaction potential

$$
U(x)=\frac{e^{2}}{|x|+R}-\frac{e^{2}}{\sqrt{x^{2}+4 D^{2}}}
$$

between electrons on the dot is short-range. For $R \ll D$ the capacitance per unit length of the
CNT is $c_{d}=1 / 2 \ln (2 D / R)$. Actually, even without the screening gate, i.e., at $D=\infty, \rho_{d}(x)$ is nearly uniform, except near the ends 13, because the potential $U(x) \propto|x|^{-1}$ is only marginally long-range in $1 \mathrm{D}$.

Let us now discuss the linear charge density $\rho_{t}(z)$ of the tip. Interestingly, it also becomes uniform [14] if $h$ and $D$ are both small and the tip is sharp, $\theta \ll 1$. In this case $w(\zeta)$ is a slowly varying function; therefore, a segment of the tip at height $z$ above the substrate is similar to a piece of thin wire of radius $w(z-h)$. The distance to the screening gate for this segment is $z+D$. A quick estimate of the capacitance of the tip per unit length $c_{t}$ can be obtained from the formula for $c_{d}$ by replacing $R \rightarrow w(z-h) \simeq(z-h) \theta$ and $D \rightarrow z+D$, i.e.,

$$
c_{t} \simeq \frac{1}{2 \ln [2(z+D) / w(z-h)]} \simeq \frac{1}{2 \ln (2 / \theta)} .
$$

This result is confirmed by a formal perturbation theory that yields $\rho_{t}(z)$ as a power series of the small parameter $1 /|\ln \theta|$, similar to Ref. [13]. In the leading order we obtain $(\zeta \equiv z-h)$

$$
\frac{1}{\rho_{t}}=\ln \left[\frac{4(L-\zeta) \zeta}{L+\zeta+2 h+2 D} \frac{\zeta+2 h+2 D}{w^{2}(\zeta)}\right] .
$$

This formula applies at all $\zeta$ except very near the ends of the tip, $\zeta=0, L$. We tested it by solving the electrostatic problem for the tip and the gate numerically. An excellent agreement was reached for, e.g., $\theta=0.1 \approx 6^{\circ}$ and $h=30 \mathrm{~nm}$, which are not too difficult to achieve experimentally [8]. For such $\theta$ and $h$ the nonuniformity of $\rho_{t}$ within the important region $0<\zeta<2 D$ (see below) is about $15 \%$ for $D=30-200 \mathrm{~nm}$. These are the parameters adoped in our subsequent simulations described shortly below.

The approximate uniformity of $\rho_{t}$ entails a simple model for the potential $U_{t}(\mathbf{r})$ induced by 
the tip on the substrate. This potential is created both by the tip itself and by its oppositely charged image [Fig. 2(a)]. It is convenient to move the latter into the $z>0$ half-space by reflection [Fig. 2(b)]. As a result, the two charge densities cancel each other almost everywhere. The important uncompensated piece is a uniformly charged rod of length $2 D$ [Fig. [2(c)]. The potential induced by this rod is

$$
U_{t}=c_{t} V_{t}\left(\sinh ^{-1} \frac{h+2 D}{\Delta r}-\sinh ^{-1} \frac{h}{\Delta r}\right),
$$

where $\Delta r=\left|\mathbf{r}-\mathbf{r}_{t}\right|$ is the in-plane distance from the tip and $V_{t}$ is the tips's voltage. In particular, for a thin dielectric substrate, $U_{t}$ has the Coulomb form,

$$
U_{t}(\mathbf{r})=Q / \sqrt{\Delta r^{2}+h^{2}}, \quad D \ll h .
$$

In this limit the effect of tip reduces to that of a point charge $Q=D V_{t} / \ln (2 / \theta)$ positioned at height $h$ above the substrate [Fig. 2(c)]. Clearly, $h$ sets the range of the tip-dot interaction and is thus the sole geometric parameter that controls the SGM spatial resolution (Res).

As $D$ increases, the resolution degrades. The following estimate can be derived:

$$
\operatorname{Res} \sim \min \{h+D, \sqrt{b h}\},
$$

where $b \gg h$ is the half-length of the quantum dot probed by the SGM. The reason why $b$ enters at large $D$ is the slow decay of $U_{t}$ with $\Delta r$. In this case Res is determined not by the behavior of $U_{t}$ itself but by that of its variation across the system, $\Delta U_{t}(\mathbf{r})=U_{t}(\mathbf{r})-U_{t}(b, 0)$. Indeed, an additive constant in the dot-tip interaction energy has no effect on spatial resolution. A rough estimate of Res is then the value of $\Delta r$ at which $\Delta U_{t}$ drops to a half of its maximum value at

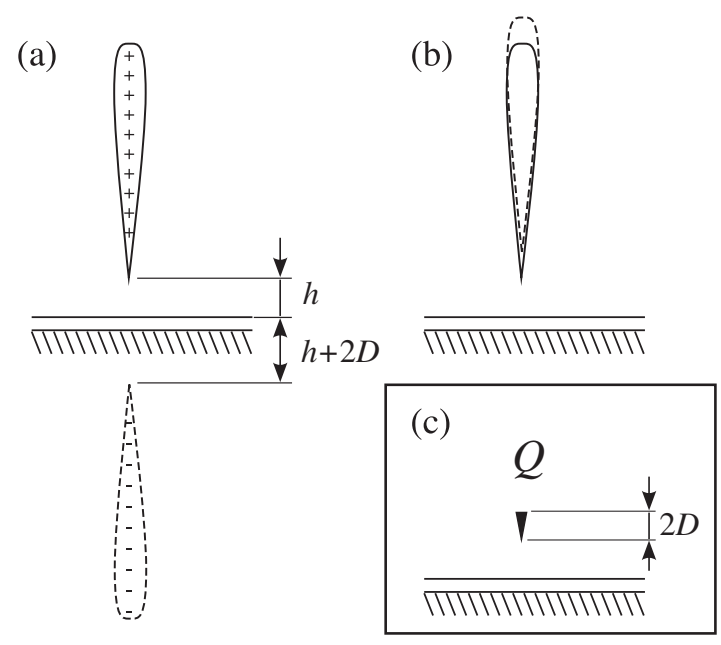

Figure 2: Geometrical construction that enables one to replace a long tip by a uniformly charged rod of a much smaller length $2 D$ (see main text).

$\Delta r=0$. Thus, in the case of a thick substrate, $D \gg b$, where Eq. (4) yields

$$
\Delta U_{t} \simeq c_{t} V_{t} \ln \left(\frac{b}{\sqrt{\Delta r^{2}+h^{2}}+h}\right), \quad r_{t} \ll b,
$$

the second expression in Eq. (6) is recovered.

Note that the actual resolution of the SGM depends on temperature. At low $T$ Coulomb blockade spectroscopy enables one to detect very small variations of the energy of the system as a function of $\mathbf{r}_{t}$. Hence, the spatial resolution also improves. Nevertheless, outperforming the estimate (6) by more than a factor of two or so would probably require impractically low $T$.

The substrates used in the current SGM experiments commonly have the effective thickness of $D \sim 50 \mathrm{~nm}$ [9], which is not much larger than a typical $h$. Therefore, Eq. (5) is acceptable as a first approximation. This also explains why an analogous model for the tip - a small metallic 
sphere - postulated previously [5] 7] was in a reasonable agreement with the experiment.

Armed with Eq. (4), we now turn to the computation of representative SGM patterns. The conductance measured in an SGM experiment is directly related to the tip-dot capacitance $C_{d t}$. When the preferred dot charge $Q_{d}=C_{d t} V_{t}+$ const is a half-integer multiple of $e$, the Coulomb blockade is lifted and the conductance peak is measured [15]. The problem reduces to calculation of $C_{d t}\left(\mathbf{r}_{t}\right)$. Let us first discuss the case of a metallic CNT where it can be done analytically.

The key idea is to treat the inverse selfcapacitances of the dot and the tip, $\ln (2 D / R)$ and $\ln (2 / \theta)$, respectively, as large parameters. Then $C_{d t}$ can be computed by a perturbation theory. In the leading order one needs to integrate the product $U_{t}(x) \rho_{d}(x)$ over the length of the dot. This gives the tip-dot interaction energy from which $C_{d t}$ can be extracted. In the simplest case $D \ll h$ one can use Eq. (5) to get

$$
C_{d t} \simeq-\frac{D \ln \left(\frac{x_{t}+b+\sqrt{\left(x_{t}+b\right)^{2}+y_{t}^{2}+h^{2}}}{x_{t}-b+\sqrt{\left(x_{t}-b\right)^{2}+y_{t}^{2}+h^{2}}}\right)}{2 \ln (2 / \theta) \ln (2 D / R)}
$$

In deriving this equation, additional screening by source and drain leads was neglected. This is legitimate away from the leads, at $b-\left|x_{t}\right| \gg$ $D, h_{l}$, where $h_{l}$ is the height by which the leads rise above the substrate.

The contours of $Q_{d}\left(\mathbf{r}_{t}\right)=C_{d t} V_{t}=(N+$ $1 / 2) e=$ const computed according to Eq. (8) for typical experimental parameters are presented in Fig. 3. They are oval-shaped near the CNT and become nearly circular at larger $N$, as in experiment 4. To verify our results for $C_{d t}$ quantitatively, we compared them with the output of the numerically exact capacitance calculator FASTCAP [16]. For parameters specified in Fig. 3] and

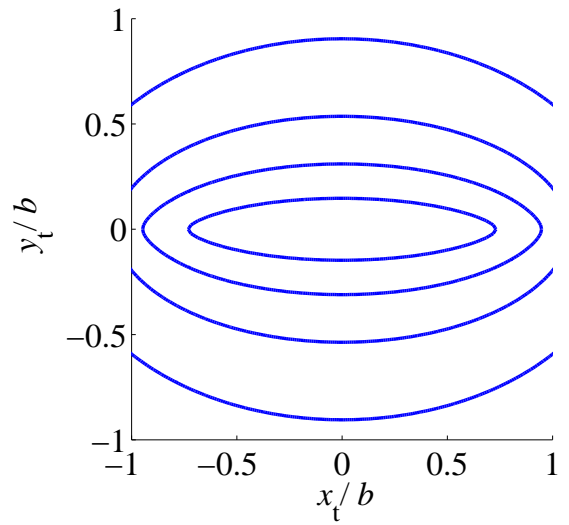

Figure 3: High-conductance contours $C_{d t} V_{t}=$ $(N+1 / 2) e=$ const as function of the tip position for a metallic CNT. $N$ runs through a set of consequtive integers. Parameters: $b=250 \mathrm{~nm}$, $R=2 \mathrm{~nm}, D=30 \mathrm{~nm}$, and $V_{t}=0.3 \mathrm{~V}$.

the tip of length $L=10 \mu \mathrm{m}$ we found a $5 \%$ agreement, which is gratifying given the simplicity of our approach and the approximations made.

Classical electrostatics we used thus far is valid for a metallic nanotube where the average interelectron separation $a$ is small. A more interesting question is whether the SGM can help us to see effects beyond continuum electrostatics, for example: Can SGM image individual electrons?

As explained earlier, Res is of the order of the height $h$ of the tip above the substrate, typically, a few tens of $\mathrm{nm}$. This is insufficient for resolving individual electrons in a metallic CNT. On the other hand, in a semiconducting CNT the mean inter-particle separation $a=20-30 \mathrm{~nm}$ can be achieved. In such CNTs electrons (or holes) can be added to the conduction (valence) band one by one starting from zero [17. Since the typical size of a CNT quantum dot is a fraction of a micron, it would contain only a few charge carriers altogether. In this regime the 
electrons form a Wigner molecule: a state where they are highly localized near the classical equilibrium positions. The Wigner-molecule approximation is accurate if $r_{s} \equiv a / 2 a_{B}>4$, [18, 19] where $a_{B}$ is the effective Bohr radius. At large $r_{s}$ the charge distribution of each electron can be approximated by a Gaussian with the standard deviation $w \approx 0.52 a_{B}\left(a / a_{B}\right)^{3 / 4}$. For example, for $a \approx 40 \mathrm{~nm}$ (as in Fig. 4) and $a_{B} \sim 1.4 \mathrm{~nm}$ (as in the experiment [17]) we get $w \approx 6.4 a_{B}$, in agreement with numerical simulations of Wigner molecules [18.

Suppose Res $<a$, then as the tip moves along the dot, it interacts primarily with the nearest electron, and so continuum electrostatics is no longer valid. Instead, one expects a significant oscillatory modulation of the tip-dot coupling: maxima when the (repulsive) tip is positioned directly above an electron and minima when it is between two adjacent electrons. Such variations may be detectable by examining the spacing of the conductance peaks.

To verify these qualitative ideas we carried out a series of numerical simulations. The Hamiltonian of the system $H$ was taken to be

$$
\begin{aligned}
& H=\sum_{1 \leq i<j \leq N} U\left(x_{i}-x_{j}\right)+\sum_{i} e U_{t}\left(x_{i}\right)+H_{\text {con }}, \\
& H_{\text {con }}=\sum_{i=1}^{N} e\left[\phi_{\text {con }}\left(x_{i}\right)+V_{g}\right]
\end{aligned}
$$

where electron-electron and electron-tip interactions are given by Eqs. (11) and (4), $V_{g}$ is the gate voltage relative to the that of the leads, and $\phi_{\text {con }}(x)$ is the confinement potential due to contact potential difference between the leads and the CNT. Following Ref. [17, we chose $\phi_{\text {con }}(x)=(k / 2)\left(x^{2}-b^{2}\right)$, with the coefficient $k$ close to the number estimated in that paper. The ground-state energy $E_{N}$ and the electron
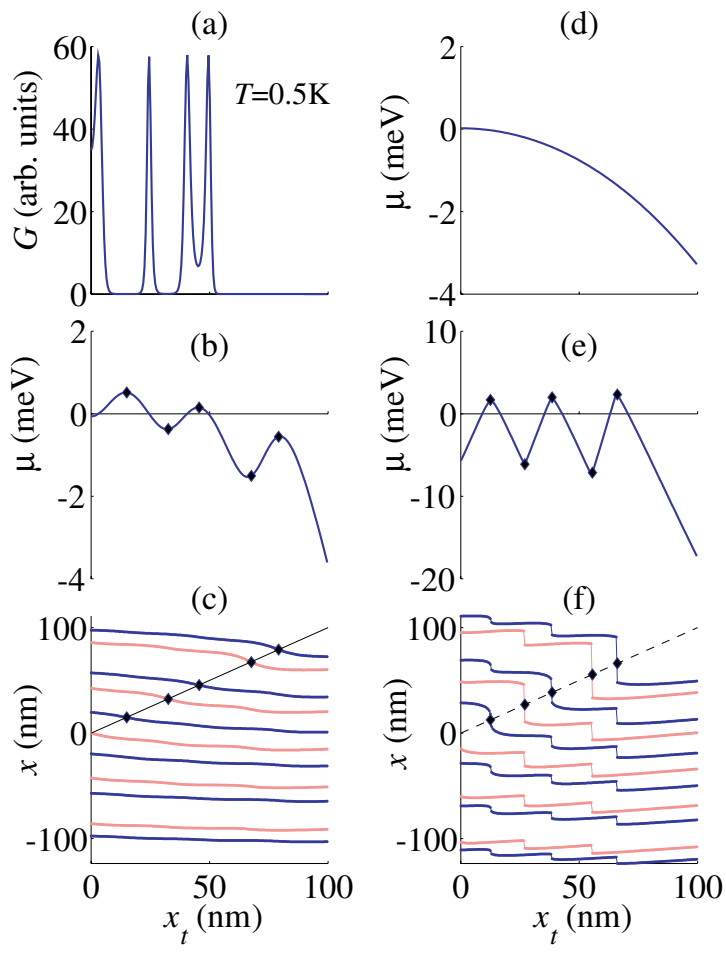

Figure 4: (Color online) Results of numerical simulations for SGM of Wigner molecules. (a) Conductance [estimated per Eq. (10)] (b) chemical potential, and (c) ground-state electron positions for $h=30 \mathrm{~nm}, D=200 \mathrm{~nm}$, and $V_{t}=$ $-0.1 \mathrm{~V}$. In plot (c) every other curve from the top is for the six-electron molecule, the rest are for the five-electron state. At a higher tip voltage, $V_{t}=-0.25 \mathrm{~V}$, plot (c) evolves into (f) and plot (b) into (e). At a larger tip height, $h=90 \mathrm{~nm}$, graph (b) evolves into (d). Diamonds correspond to $x_{t}$ at which the tip is directly above one of the electrons. 
positions $x_{j}$ were found numerically for a dense grid of tip coordinates on the interval $0<x_{t}<b$. Figure 4 shows the results. Unlike Fig. 3 here we focus on line scans where the tip stays directly above the CNT, i.e., at $y_{t}=0$.

At low $T$ there are only two important charge states to consider, $Q_{d}=N e$ and $Q_{d}=(N+1) e$. In the simulations the gate voltage $V_{g}$ was adjusted to have $N=5$. The dependence of the electron positions on $x_{t}$ in the two competing configurations is shown in Fig. 4(c) for the relatively low voltage of $V_{t}=-0.1 \mathrm{~V}$. The displacements of the electrons by the tip are rather small, so here the tip is a weakly invasive probe. Shown by diamonds are the tip positions where it is directly above an electron. The chemical potential $\mu \equiv E_{N+1}-E_{N}$ for the same SGM scan is plotted in Fig. 4(b). It exhibits the expected spatial variations: maxima and minima near $x_{t}$ 's of the diamonds. At the intersection of the $\mu\left(x_{t}\right)$ curve with the horizontal line $\mu=0$ the two charged states become degenerate. At such points the conductance $G$ through the dot has peaks. The calculation of the shape of these peaks is beyond the scope of this work. However, for illustrative purposes, in Fig. 4(a), we plot the expression

$$
G=\frac{1}{2 T R_{s d}} \frac{\mu}{\sinh (\mu / T)},
$$

which holds for large metallic dots [15. Parameter $R_{s d}$ here denotes the sum of the tunneling resistances at the source and the drain leads.

According to the discussion above, imaging the electron positions amounts to finding the maxima and minima of the chemical potential. To achieve that in experiment one would need to repeat SGM scans at different $V_{g}$. Let $\mu\left(x_{t}\right)$ be the chemical potential at some initial gate voltage $V_{g}^{*}$. At a different $V_{g}$, the chemical potential changes by a constant, i.e., the curve in
Fig. 4(b) shifts up or down as a whole. The conductance peaks are found at the roots of the equation $\mu\left(x_{t}\right)=e V_{g}^{*}-e V_{g}$. Based on this relation, the entire curve of $\mu\left(x_{t}\right)$ can be deduced by tracking the peak positions as a function of $V_{g}$.

As the tip's voltage increases, it perturbs the system stronger, see Fig. 廿 (f). Here we focus on the case of a repulsive tip, which expels electrons from underneath itself. This creates a double quantum dot: Dot 1 in front of the tip and Dot 2 behind it [regions above and below the dashed line in Fig. 4(d), respectively]. As the tip moves, the following sudden changes in the double-dot system take place: (1) an electron is expelled from Dot 1 into the lead, which occurs when $\mu<$ 0 changes to $\mu>0,(2)$ another electron enters Dot 2 from the opposite lead, at the points where $\mu>0$ changes to $\mu<0$, and (3) an electron jumps between Dots 1 and 2, at the positions labelled by diamonds in Fig. 目(f). Under the assumptions that contact resistance $R_{s d}$ is higher than the inter-dot tunneling resistance and the temperature is low enough, the peaks in total conductance $G$ coincide 20 with events (1) and (2), i.e., the points $\mu=0$.

The strongly invasive SGM can be easily distinguished from a weakly invasive one experimentally. First, in the invasive regime $\mu\left(x_{t}\right)$ curve has cusps [Fig. 廿4(e)] whereas in the weakly

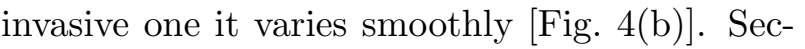
ond, in the double-dot case the peak heights of the conductance have a strong modulation (beating pattern) 21] as a function of $x_{t}$ and $V_{g}$. The highest $G$ is achieved if the Coulomb blockade in the two dots is lifted simultaneously. This can be realized by tuning $V_{g}$ to some special values at which cusps of $\mu\left(x_{t}\right)$ touch the $\mu=0$ line. We do not attempt to discuss $G$ in any more detail because its calculation for the double-dot is a complicated problem [20. Equation (10) be- 
comes a poor approximation in this regime.

The effect of the tip height on spatial resolution is demonstrated in Fig. 4(d): for $h=90 \mathrm{~nm}$ the oscillations in $\mu\left(x_{t}\right)$ are completely obliterated. This confirms that the resolution limit strongly depends on $h$. In contrast, having a rather large $D=200 \mathrm{~nm}$ does not degrade the resolution much. It may be surprising at first but, in fact, Eq. (6) gives Res $\sim 55 \mathrm{~nm}$ (using $b=\max \left|x_{j}\right| \approx 100 \mathrm{~nm}$ ), which is only slightly larger than the mean electron spacing $a \approx 40 \mathrm{~nm}$. Note also that a rather low temperature, $T=0.5 \mathrm{~K}$, is chosen.

Finally, let us discuss the results for a twodimensional SGM scan, Fig. 5. We once again see contours of $G$, akin to Fig. 3 but with striking differences. One of the contours contains pronounced oscillatory features — "wiggles". Another contour (closest to the CNT) has been broken into three disconnected pieces, as though the amplitude of the corresponding wiggles exceeded this contour's original width. Similar to the 1D scans of Fig. 4 such effects originate from discreteness of the electron charge that causes modulation of the chemical potential as the tip passes by individual electrons. In general, the contour with $N$ wiggles separates the interior region of $N-1$ electrons from the exterior one with $N$ electrons in the ground-state. The lack of wiggles on more distant contours is explained by noting that the spatial resolution is now set by $\sqrt{h^{2}+y_{t}^{2}}$, i.e., it worsens as the tip is moved laterally away from the dot. At large $y_{t}$ continuum electrostatics applies, and so the distant $G$-contours are similar to those of Fig. 3.

To assess how stable the predicted pattern is against thermal smearing, $G$ in Fig. 5 is computed for $T=15 \mathrm{~K}$. For simplicity, the calculation is done based on Eq. (10) although this equation is a poor approximation in this case.

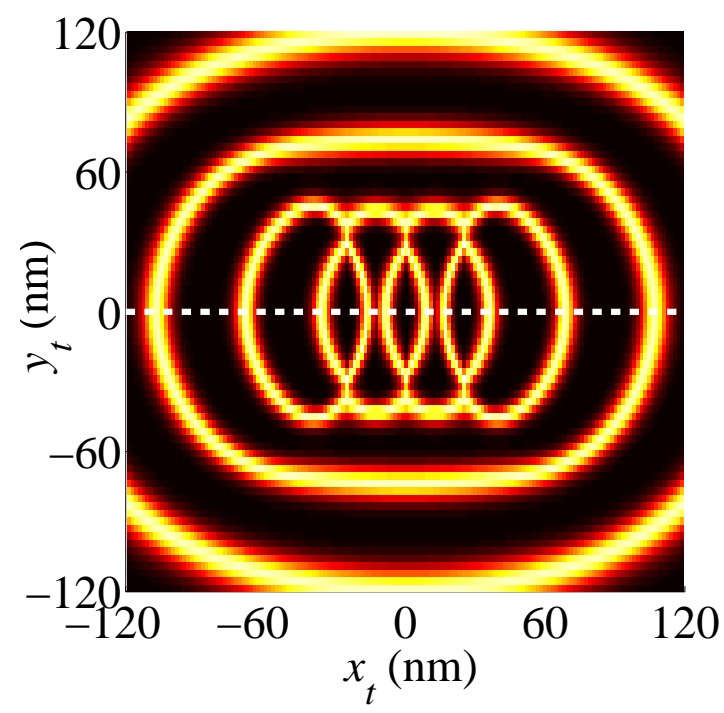

Figure 5: (Color online) Contours of high conductance in a low- $T$ two-dimensional SGM scan. Simulation parameters: $D=30 \mathrm{~nm}, h=30 \mathrm{~nm}$, $V_{t}=-0.4 \mathrm{~V}, T=15 \mathrm{~K}$.

In particular, it gives a constant intensity along the contours of high $G$ whereas in reality it is expected to have strong variations. A more accurate calculation of $G$ is deferred for future work.

In conclusion, we have studied electrostatics of a prototypical SGM experiment in which a CNT quantum dot is probed by a sharp metallic tip. We developed an analytical model for the coupling between the tip and charges on the sample plane and verified it by numerical simulations. Our model explains why the resolution is controlled primarily by the tip-dot distance (the height above the substrate and the lateral distance away from the CNT) rather than by much larger overall dimensions of the tip and the dot. We predicted that the SGM of a semiconducting $\mathrm{CNT}$ in the regime of strong electron-electron interaction may be able to detect positions of 
individual electrons forming a Wigner molecule. Lastly, the tip can act as either a weakly or a strongly invasive probe, and so the SGM can be employed both for single-electron imaging and for nano-manipulation.

This work is supported by UCSD ASCR and C.\& W. Hellman Fund. We thank S. Kalinin, P. McEuen, and J. Zhu for valuable comments.

\section{References}

[1] S. J. Tans and C. Dekker, Nature (London) 404, 834 (2000).

[2] A. Bachtold, M. S. Fuhrer, S. Plyasunov, M. Forero, E. H. Anderson, A. Zettl, and P. L. McEuen, Phys. Rev. Lett. 84, 6082 (2000).

[3] M. Bockrath, W. Liang, D. Bozovic, J. H. Hafner, C. M. Lieber, M. Tinkham, and H. Park, Science 291, 283 (2000).

[4] M. Woodside, $\mathrm{PhD}$ thesis, University of California, Berkeley, 2001; M. T. Woodside and P. L. McEuen, Nature (London) 296, 1098 (2002).

[5] M. Freitag, S. V. Kalinin, D. A. Bonnell, and A. T. Johnson, Phys. Rev. Lett. 89, 216801 (2002).

[6] C. Staii, A. T. Johnson, R. Shao, and D. A. Bonnell, NanoLett. 5, 893 (2005).

[7] V. Meunier, S. V. Kalinin, J. Shin, A. P. Baddorf, and R. J. Harrison, Phys. Rev. Lett. 93, 246801 (2004);

[8] Most commonly, $\theta \sim 0.25$ and $l \sim 20 \mathrm{~nm}$ but much smaller values are feasible with ion-milled tips.
[9] If the substrate has the dielectric constant $\epsilon \neq 1$, its thickness is effectively reduced by $\epsilon$, e.g., for the geometrical thickness of $200 \mathrm{~nm}$ and $\epsilon=3.9$ of $\mathrm{SiO}_{2}, D \approx 50 \mathrm{~nm}$.

[10] P. Pomorski, L. Pastewka, Ch. Roland, H. Guo, and J. Wang, Phys. Rev. B 69, 115418 (2004).

[11] M. Paillet, P. Poncharal, and A. Zahab, Phys. Rev. Lett. 94, 1868011 (2005), and references therein.

[12] A. Svizhenko and M. P. Anantram, Phys. Rev. B 72, 85430 (2005).

[13] J. D. Jackson, Am. J. Phys. 68, 789 (2000).

[14] S. Belaidi, P. Girard, and G. Leveque, J. Appl. Phys. 81, 1023 (1997).

[15] Single-Charge Tunneling, edited by H. Grabert and M. H. Devoret (Plenum, New York, 1992).

[16] FastCap, Research Laboratory of Electronics, MIT. Available at http://www.rle.mit.edu.

[17] P. Jarillo-Herrero, S. Sapmaz, C. Dekker, L. P. Kouwenhoven, and H. S. J. van der Zant, Nature (London) 429, 389 (2004).

[18] For a review, see S. M. Reimann and M. Manninen, Rev. Mod. Phys. 74, 1283 (2002).

[19] M. M. Fogler and E. Pivovarov, Phys. Rev. B 72, 195344 (2005).

[20] K. A. Matveev, L. I. Glazman, and H. U. Baranger, Phys. Rev. B 54, 5637 (1996). 
[21] W. G. van der Wiel, S. De Franceschi, J. M. Elzerman, T. Fujisawa, S. Tarucha, and L. P. Kouwenhoven, Rev. Mod. Phys. 75, 1 (2003). 